\title{
Cost-effectiveness of oral semaglutide added to current antihyperglycemic treatment for type 2 diabetes
}

\author{
Gregory F Guzauskas, MSPH, PhD; David M Rind, MD, MSc; Katherine Fazioli; \\ Richard H Chapman, PhD, MS; Steven D Pearson, MD, MSc; and Ryan N Hansen, PharmD, PhD
}

\section{What is already known about this subject}

- The Institute for Clinical and Economic Review (ICER) performed a previous systematic literature review and cost-effectiveness analysis to evaluate the health and economic outcomes of oral semaglutide, the first oral formulation of a glucagonlike peptide 1 receptor agonist approved in the United States, for the treatment of type 2 diabetes mellitus.

- We previously found that semaglutide (oral and injectable) reduced the risk of 3-point major adverse cardiovascular event more than sitagliptin; no significant differences were found between semaglutide and liraglutide or empagliflozin.

- The previous economic analysis showed that oral semaglutide had the highest life-years (LYs) and qualityadjusted life-years (QALYs) gained among comparators and, using a placeholder cost for oral semaglutide, was cost saving compared with liraglutide, cost-effective compared with background therapy alone and sitagliptin, but cost prohibitive compared with empagliflozin.

\section{What this study adds}

- Compared with the original ICER review, the actual wholesale acquisition cost for oral semaglutide was incorporated into the analysis, and all drug costs have been updated to May 2020 estimates.

- Oral semaglutide use resulted in better outcomes (e.g., LYs and QALYs) than background treatment alone or sitagliptin and similar outcomes to liraglutide or empagliflozin.

- Oral semaglutide was estimated to be cost-effective compared with liraglutide and to have incremental cost-effectiveness ratios between $\$ 100,000$ and $\$ 150,000$ per QALY versus sitagliptin and background therapy alone, but it did not meet these thresholds compared with empagliflozin.

\section{ABSTRACT}

BACKGROUND: Oral semaglutide is the first oral formulation of a glucagon-like peptide 1 (GLP-1) receptor agonist to be approved in the United States for glycemic control in people with type 2 diabetes mellitus (T2DM). While oral semaglutide is not indicated for reduction of cardiovascular event risk, its label does include evidence of no increase in cardiovascular risk in people who received oral semaglutide.

\begin{abstract}
Author affiliations
Gregory F Guzauskas, MSPH, PhD, and Ryan $\mathrm{N}$ Hansen, PharmD, PhD, The Comparative Health Outcomes, Policy, and Economics (CHOICE) Institute, Department of Pharmacy, University of Washington, Seattle. David M Rind, MD, MSc; Katherine Fazioli; Richard H Chapman, PhD, MS; and Steven D Pearson, MD, MSc, Institute for Clinical and Economic Review (ICER), Boston, MA.
\end{abstract}

\section{AUTHOR CORRESPONDENCE:}

Ryan N Hansen, 206.543.2402;

rhansen@uw.edu

J Manag Care Spec Pharm. 2021;27(4):455-68

Copyright $\odot 2021$, Academy of Managed Care Pharmacy. All rights reserved.

OBJECTIVE: To estimate the incremental value of oral semaglutide added to existing antihyperglycemic treatment for people with T2DM with additional risk for cardiovascular disease. 
METHODS: We estimated the lifetime cost-effectiveness of oral semaglutide added to current antihyperglycemic treatment for T2DM using a microsimulation model based primarily on the UK Prospective Diabetes Study (UKPDS) Outcomes Model 2 (OM2) equations. Oral semaglutide added to current antihyperglycemic treatment was separately compared with (a) ongoing background antihyperglycemic treatment, (b) sitagliptin, (c) empagliflozin, and (d) liraglutide. Comparators sitagliptin, empagliflozin, and liraglutide were added to ongoing antihyperglycemic treatment. We applied hazard ratios derived from a network meta-analysis for cardiovascular and renal outcomes to the UKPDS OM2 estimated baseline rates. Health state utilities and costs were derived from the published literature. We estimated total costs, life-years (LYs), quality-adjusted life-years (QALYs), clinical events, and cost per major adverse cardiovascular event (MACE) avoided, over a lifetime time horizon using discount rates of $3 \%$ for costs and outcomes.

RESULTS: The lifetime total cost for people treated with oral semaglutide was $\$ 311,300$, with costs for the other comparators ranging from $\$ 262,800$ (background treatment alone) to $\$ 287,800$ (liraglutide). Oral semaglutide resulted in the fewest MACE, including the fewest cardiovascular deaths. Among the 5 modeled treatment strategies, oral semaglutide had the highest LYs gained (8.43 vs. 7.76 [background treatment alone] to 8.29 [empagliflozin and liraglutide]) and the highest QALYs gained (4.11 vs. 3.70 [background treatment alone] to 4.03 [empagliflozin]). Oral semaglutide would likely be considered cost-effective compared with liraglutide (incremental cost-effectiveness ratio $[I C E R]=\$ 40,100)$, and moderately cost-effective versus background treatment alone $([\mathrm{ICER}]=\$ 117,500 / \mathrm{QALY})$ and sitagliptin $($ ICER $=\$ 145,200 / Q A L Y)$. The ICER for oral semaglutide compared with empagliflozin was approximately $\$ 458,400$ per QALY.

CONCLUSIONS: As modeled, oral semaglutide as an add-on therapy to background antihyperglycemic treatment produced incremental benefits in MACE avoided, along with greater QALYs compared with background antihyperglycemic treatment alone. Oral semaglutide use resulted in better outcomes than background treatment alone or sitagliptin, and similar outcomes to liraglutide or empagliflozin with overlapping 95\% confidence ranges for QALYs. Oral semaglutide was estimated to be cost-effective compared with liraglutide and to have incremental cost-effectiveness ratios between $\$ 100,000$ and $\$ 150,000$ per QALY versus sitagliptin and background therapy alone, but it did not meet these thresholds compared with empagliflozin.

The vast majority of the 30 million people with diabetes mellitus in the United States have type 2 diabetes mellitus (T2DM). ${ }^{1}$ People with diabetes experience elevated blood glucose and are at increased risk of macrovascular and microvascular complications. These complications frequently include damage to the eyes and kidneys, as well as myocardial infarction (MI), stroke, limb ischemia, and increased risk of cardiovascular death. ${ }^{2}$ Improving blood glucose control can reduce the risk of microvascular complications and, particularly in individuals newly diagnosed with diabetes, may also reduce the risk of macrovascular complications. ${ }^{3}$

The overall U.S estimated cost of diabetes (medical costs and productivity loss) was approximately \$327 billion in 2018. ${ }^{4}$ At the individual level, diabetes also takes a significant financial toll. According to the Centers for Disease Control and Prevention, a quarter of people with diabetes have asked their health care providers to prescribe a lowercost medication, and $13 \%$ were nonadherent because of out-of-pocket costs. ${ }^{5}$

T2DM management should be tailored to the individual patient and typically begins with a foundation of medical nutrition therapy and physical activity (lifestyle changes). While these changes are sufficient to achieve adequate glycemic control in some individuals, frequently antihyperglycemic medications are added to achieve and sustain glycemic control. ${ }^{2,6}$ Pharmacotherapy typically follows a staged approach, with metformin as the preferred first-line medication option, followed by a variety of augmentation strategies. ${ }^{2,6}$ Metformin has a favorable safety profile in that it does not increase weight or the risk of hypoglycemia (low blood glucose) when used as a single agent., ${ }^{2,6}$ Additional pharmacotherapy options include oral (e.g., sulfonylureas, thiazolidinediones, sodium-glucose cotransporter 2 [SGLT-2] inhibitors, and dipeptidyl peptidase-4 [DPP-4] inhibitors) and injectable medications (e.g., glucagon-like peptide 1 [GLP-1] receptor agonists and insulin). ${ }^{2,6}$

GLP-1 receptor agonists have been available as injectable medications for several years in the United States, but in September 2019, the first oral GLP-1 receptor agonist, semaglutide (Rybelsus, Novo Nordisk), was approved for the treatment of adults with T2DM. The injectable form of semaglutide has been available in the United States since 2017. The manufacturer may also seek approval from the U.S. Food and Drug Administration of a labeled indication to reduce major cardiovascular events in adults with T2DM and established cardiovascular disease. ${ }^{8}$ Since oral semaglutide is the first oral formulation of a GLP-1 receptor agonist to be approved in the United States, we sought to estimate its incremental value to the U.S. health care system.

\section{Methods}

\section{APPROACH}

The primary aim of this analysis was to estimate the lifetime cost-effectiveness of oral semaglutide added to current antihyperglycemic treatment for T2DM, using a decision analytic model. Oral semaglutide (14 mg daily) added to 


\section{FIGURE 1 Microsimulation Model}

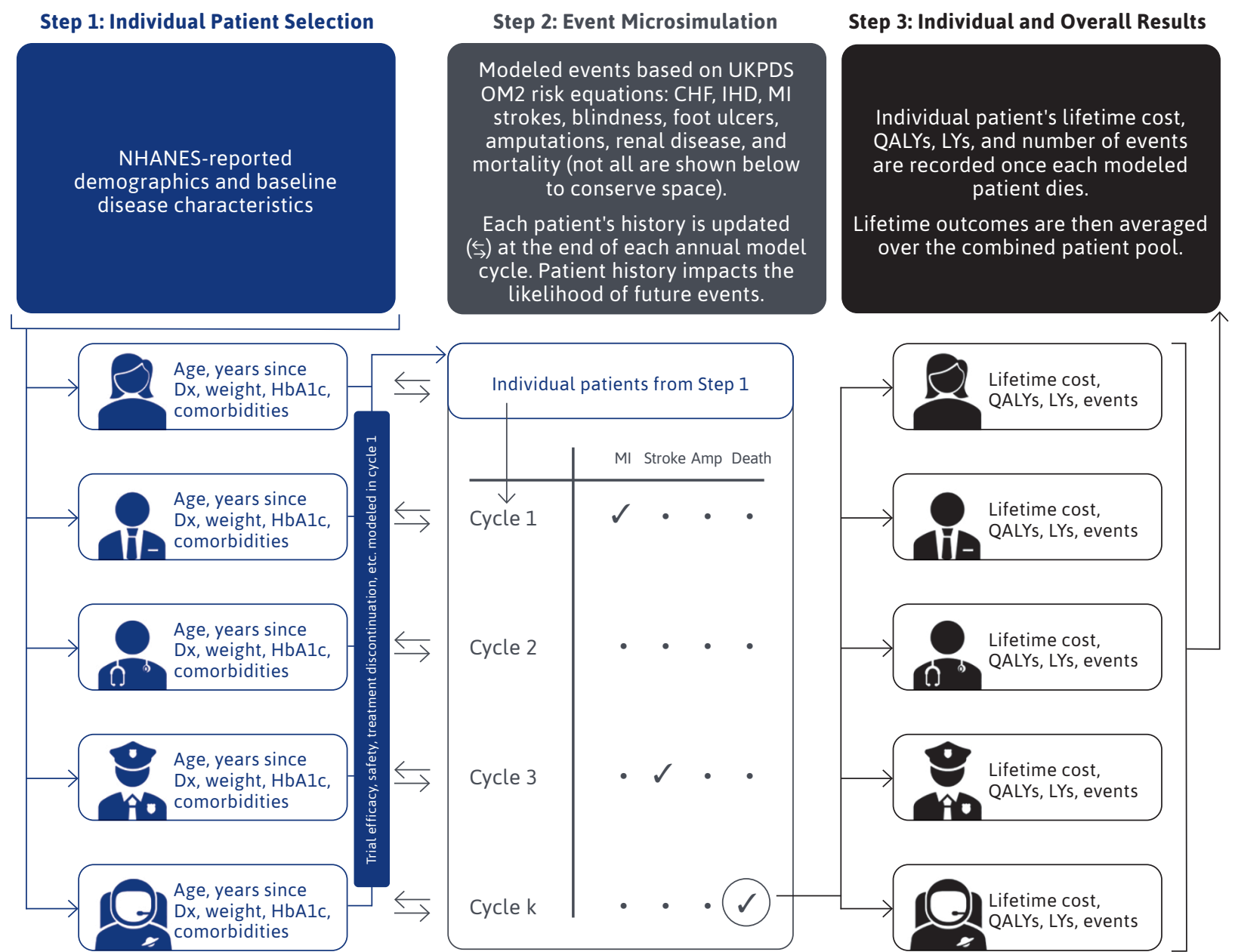

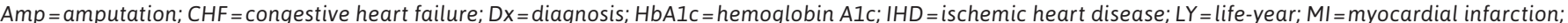
NHANES=National Health and Nutrition Examination Survey; QALY=quality-adjusted life-year; UKPDS OM2 = United Kingdom Prospective Diabetes Study Outcomes Model 2.

current antihyperglycemic treatment was separately compared with 4 modeled comparators: (1) empagliflozin (an SGLT-2 inhibitor, $10 \mathrm{mg}$ or $25 \mathrm{mg}$ daily); (2) liraglutide (an injectable GLP-1 receptor agonist, $1.8 \mathrm{mg}$ daily); (3) sitagliptin (a DPP-4 inhibitor, $100 \mathrm{mg}$ daily); and (4) ongoing background antihyperglycemic treatment (e.g., metformin with or without sulfonylureas). Similar to oral semaglutide, comparators empagliflozin, liraglutide, and sitagliptin are added to ongoing antihyperglycemic treatment. These comparators were selected based on the ability to create network meta-analysis comparisons with oral semaglutide. The base-case analysis used a health care sector perspective (i.e., direct medical care costs only from all payment sources) and a lifetime time horizon. All costs and outcomes were discounted at 3\% per year. The model was developed in Microsoft Excel for Office 365, version 1911 (Microsoft Corp., Redmond, WA).

\section{POPULATION}

We modeled adults with T2DM with inadequate glycemic control despite being currently treated with antihyperglycemic agents. We used a U.S. population of people with T2DM, drawing patient-level data from the National Health and Nutrition Examination Survey (NHANES), which surveys approximately 5,000 people across the United States 
each year. ${ }^{9}$ A cohort with self-reported diabetes and hemoglobin A1c $\geq 7$ from NHANES 2013-14 and 2015-16 surveys $(\mathrm{n}=362)$ served as the population for our microsimulations (Supplementary Table 1, available in online article).

\section{CARDIOVASCULAR AND RENAL OUTCOMES DATA SYNTHESIS}

We quantitatively synthesized data informing the comparison of oral semaglutide and comparators of interest on cardiovascular and renal benefits in network meta-analyses (NMAs). ${ }^{10}$ We analyzed 3-point major adverse cardiovascular events (MACE; a composite of cardiovascular death, nonfatal MI, or nonfatal stroke); hospitalization for heart failure (HF); and new or worsening nephropathy. Data from the cardiovascular outcomes trials (CVOTs) of oral semaglutide (PIONEER 6) and injectable semaglutide (SUSTAIN 6) informed the cardiovascular and renal effects of semaglutide ${ }^{11,12}$; we then conducted a random effects meta-analysis of treatment effects from these 2 trials using the metafor package in R. ${ }^{13}$ We subsequently performed NMAs using the gemtc package in $\mathrm{R}^{14}$ to synthesize the results from the semaglutide meta-analysis with results from the CVOTs of the comparators to obtain indirect estimates of outcomes for semaglutide (as a molecule) relative to each comparator. ${ }^{15-17}$

\section{MICROSIMULATION MODEL}

We developed an individual patient-level, Monte Carlobased microsimulation of costs, quality of life, clinical events, and mortality associated with T2DM among U.S. adults diagnosed with the disease (Figure 1). Our model was adapted from a published microsimulation model, ${ }_{1}^{18}$ which used the United Kingdom Prospective Diabetes Study (UKPDS) Outcomes Model 2 (OM2). ${ }^{19}$ The UKPDS OM2 risk equations are widely used in diabetes simulation models and have been shown to accurately predict results for the population in which it was developed, as well as in other diabetes populations. ${ }^{19-21}$

The risk equations (13 T2DM complication equations and 4 mortality equations) include $\mathrm{HF}$, ischemic heart disease (IHD), first MI for females, first MI for males, subsequent MI, first stroke, subsequent stroke, blindness, foot ulcer, first amputation without prior ulcer, first amputation with prior ulcer, subsequent amputation, and end-stage renal disease (ESRD)..$^{19}$ The 4 mutually exclusive mortality risk equations were death without history of complications, death in the year of a clinical event, death in subsequent year of prior events, and death with history of clinical events. ${ }^{19}$ People were able to experience multiple and concurrent T2DM complications during each modeled year. The mortality risk equations predict that previous T2DM-related complications (except foot ulcer and blindness) increase the probability of death.

Three common modeling steps were used for each individual: (1) individual patient simulation of PIONEER trial results in the first cycle; (2) individual patient simulation of lifetime macrovascular and microvascular events and associated costs and quality of life; and (3) calculation of mean results from the pool of simulated persons' lifetime outcomes. Each person was separately modeled as receiving each comparator until they died with no further changes to background therapy, at which point the lifetime outcomes per person and per comparator were recorded.

Patients who discontinued initial treatment were assumed to transition to insulin therapy to facilitate headto-head comparator evaluations, as opposed to evaluating differences in multiple potential treatment pathways. ${ }^{22}$ After cycle 1, we assumed that individuals treated with oral semaglutide, empagliflozin, and liraglutide added insulin therapy while remaining on their current treatment if their A1c reached 8.5 or above, while sitagliptin-treated individuals were assumed to discontinue sitagliptin and transition to insulin if their A1c reached 8.5 or above.

We assumed all people entering the model had no previous history of amputations, blindness, foot ulcers, or hypoglycemia, and we independently simulated histories of atrial fibrillation and peripheral artery disease because of a lack of this information in the NHANES data. Finally, we omitted cost and disutility data for severe or serious adverse events other than hypoglycemia, since the PIONEER trials did not present adverse event data by type of event. ${ }^{23-25}$

\section{CLINICAL INPUTS}

Short-term, trial-derived efficacy estimates from the head-to-head PIONEER trials were applied in the first model cycle to each person entering the microsimulation (Table 1). ${ }^{23-25}$ These estimates included treatment-induced changes from baseline in A1c and body weight, occurrences of severe hypoglycemia, and treatment discontinuation due to adverse events. Based on trial data, oral semaglutide had greater reductions in A1c and body weight versus the other 4 comparators (with the exception of oral semaglutide and empagliflozin having similar body weight reductions) but a higher rate of treatment discontinuation due to adverse events. Discontinuation was modeled by applying trialreported discontinuation rates for each treatment at the end of cycle 1 .

We used the hazard ratios (HRs) estimated from the NMA of cardiovascular and renal outcomes that were previously described to model long-term differences in these events among comparators. ${ }^{10}$ The HRs were applied to the UKPDS 


\section{TABLE 1 Model Parameters}

\begin{tabular}{|c|c|c|c|c|c|}
\hline & Base Case & Lower & Upper & $\begin{array}{l}\text { Probabilistic } \\
\text { Distribution }\end{array}$ & Source \\
\hline \multicolumn{6}{|l|}{ PIONEER Trial Outcomes } \\
\hline \multicolumn{6}{|l|}{ Change in HbAlc (cycle 1) } \\
\hline Oral semaglutide & -1.24 & -1.48 & -0.99 & Normal & PIONEER $2,3,4^{23-25}$ \\
\hline Sitagliptin & -0.74 & -0.88 & -0.59 & Normal & PIONEER $3^{25}$ \\
\hline Empagliflozin & -0.84 & -1.00 & -0.67 & Normal & PIONEER $2^{24}$ \\
\hline Liraglutide & -0.94 & -1.12 & -0.75 & Normal & PIONEER $4^{23}$ \\
\hline Background Tx & -0.24 & -0.28 & -0.19 & Normal & PIONEER $4^{23}$ \\
\hline \multicolumn{6}{|l|}{ Change in weight (cycle 1) } \\
\hline Oral semaglutide & $-3.8 \mathrm{~kg}$ & $-4.5 \mathrm{~kg}$ & $-3.0 \mathrm{~kg}$ & Normal & PIONEER $2,3,4^{23-25}$ \\
\hline Sitagliptin & $-1.1 \mathrm{~kg}$ & $-1.3 \mathrm{~kg}$ & $-0.9 \mathrm{~kg}$ & Normal & PIONEER $3^{25}$ \\
\hline Empagliflozin & $-3.6 \mathrm{~kg}$ & $-4.3 \mathrm{~kg}$ & $-2.9 \mathrm{~kg}$ & Normal & PIONEER $2^{24}$ \\
\hline Liraglutide & $-2.5 \mathrm{~kg}$ & $-3.0 \mathrm{~kg}$ & $-2.0 \mathrm{~kg}$ & Normal & PIONEER $4^{23}$ \\
\hline Background Tx & $-0.5 \mathrm{~kg}$ & $-0.6 \mathrm{~kg}$ & $-0.4 \mathrm{~kg}$ & Normal & PIONEER $4^{23}$ \\
\hline \multicolumn{6}{|l|}{ Severe hypoglycemia (cycle 1) } \\
\hline Oral semaglutide & 0.002 & 0.001 & 0.002 & Beta & PIONEER $2,3,4^{23-25}$ \\
\hline Sitagliptin & 0.007 & 0.006 & 0.008 & Beta & PIONEER $3^{25}$ \\
\hline Empagliflozin & 0.002 & 0.001 & 0.002 & Beta & PIONEER $2^{24}$ \\
\hline Liraglutide & 0.000 & 0.000 & 0.000 & Beta & PIONEER $4^{23}$ \\
\hline Background Tx & 0.000 & 0.000 & 0.000 & Beta & PIONEER $4^{23}$ \\
\hline \multicolumn{6}{|l|}{ Treatment discontinuation (cycle 1 ) } \\
\hline Oral semaglutide & 0.111 & 0.089 & 0.133 & Beta & PIONEER $2,3,4^{23-25}$ \\
\hline Sitagliptin & 0.049 & 0.039 & 0.059 & Beta & PIONEER $3^{25}$ \\
\hline Empagliflozin & 0.046 & 0.036 & 0.055 & Beta & PIONEER $2^{24}$ \\
\hline Liraglutide & 0.094 & 0.075 & 0.112 & Beta & PIONEER $4^{23}$ \\
\hline Background Tx & 0.036 & 0.029 & 0.043 & Beta & PIONEER $4^{23}$ \\
\hline \multicolumn{6}{|l|}{ Macrovascular Hazard Ratios } \\
\hline \multicolumn{6}{|l|}{ Composite MACE } \\
\hline Oral semaglutide vs. placebo & 0.76 & 0.63 & 0.93 & Log Normal & $\mathrm{NMA}^{10}$ \\
\hline Empagliflozin vs. oral semaglutide & 1.13 & 0.89 & 1.44 & Log Normal & $\mathrm{NMA}^{10}$ \\
\hline Liraglutide vs. oral semaglutide & 1.14 & 0.91 & 1.43 & Log Normal & NMA $^{10}$ \\
\hline Sitagliptin vs. oral semaglutide & 1.30 & 1.04 & 1.63 & Log Normal & $\mathrm{NMA}^{10}$ \\
\hline \multicolumn{6}{|l|}{ Heart failure } \\
\hline Oral semaglutide vs. placebo & 1.03 & 0.76 & 1.40 & Log Normal & $\mathrm{NMA}^{10}$ \\
\hline Empagliflozin vs. oral semaglutide & 0.63 & 0.42 & 0.95 & Log Normal & $\mathrm{NMA}^{10}$ \\
\hline Liraglutide vs. oral semaglutide & 0.84 & 0.59 & 1.21 & Log Normal & $\mathrm{NMA}^{10}$ \\
\hline Sitagliptin vs. oral semaglutide & 0.97 & 0.68 & 1.40 & Log Normal & $\mathrm{NMA}^{10}$ \\
\hline
\end{tabular}




\section{TABLE 1 Model Parameters (continued)}

\begin{tabular}{|c|c|c|c|c|c|}
\hline & Base Case & Lower & Upper & $\begin{array}{l}\text { Probabilistic } \\
\text { Distribution }\end{array}$ & Source \\
\hline \multicolumn{6}{|l|}{ Macrovascular Hazard Ratios } \\
\hline \multicolumn{6}{|l|}{ Nephropathy } \\
\hline Oral semaglutide vs. placebo & 0.64 & 0.46 & 0.89 & Log Normal & $\mathrm{NMA}^{10}$ \\
\hline Empagliflozin vs. oral semaglutide & 0.95 & 0.67 & 1.35 & Log Normal & $\mathrm{NMA}^{10}$ \\
\hline Liraglutide vs. oral semaglutide & 1.22 & 0.85 & 1.75 & Log Normal & $\mathrm{NMA}^{10}$ \\
\hline Sitagliptin vs. oral semaglutide & 1.00 & 0.80 & 1.20 & Log Normal & $\mathrm{NMA}^{10}$ \\
\hline \multicolumn{6}{|l|}{ Quality of Life } \\
\hline Baseline T2DM utility & 0.800 & 0.755 & 0.845 & Beta & Shao ${ }^{26}$ \\
\hline \multicolumn{6}{|l|}{ Macrovascular complications } \\
\hline Congestive heart failure event & -0.089 & -0.132 & -0.046 & Normal & Shao 26 \\
\hline Congestive heart failure history & -0.041 & -0.061 & -0.021 & Normal & Shao ${ }^{26}$ \\
\hline Ischemic heart disease history & -0.016 & -0.026 & -0.006 & Normal & Shao 26 \\
\hline Myocardial infarction event & -0.042 & -0.073 & -0.011 & Normal & Shao 26 \\
\hline Myocardial infarction history & -0.011 & -0.023 & 0.001 & Normal & Shao 26 \\
\hline Stroke event & -0.204 & -0.273 & -0.135 & Normal & Shao ${ }^{26}$ \\
\hline Stroke history & -0.101 & -0.117 & -0.085 & Normal & Shao ${ }^{26}$ \\
\hline \multicolumn{6}{|l|}{ Microvascular complications } \\
\hline Blindness history & -0.057 & -0.075 & -0.039 & Normal & Shao $^{26}$ \\
\hline Foot ulcer event & -0.024 & -0.034 & -0.014 & Normal & Sullivan ${ }^{27}$ \\
\hline Amputation event & -0.051 & -0.108 & 0.006 & Normal & Sullivan ${ }^{27}$ \\
\hline Renal disease history & -0.024 & -0.055 & 0.007 & Normal & Shao ${ }^{26}$ \\
\hline \multicolumn{6}{|l|}{ Hypoglycemia } \\
\hline Hypoglycemia event & -0.036 & -0.056 & -0.016 & Normal & Shao $^{26}$ \\
\hline Hypoglycemia history & -0.033 & -0.055 & -0.011 & Normal & Shao ${ }^{26}$ \\
\hline Annual disutility for Tx injection & -0.054 & -0.065 & -0.043 & Normal & Shao 26 \\
\hline \multicolumn{6}{|l|}{ Clinical Costs } \\
\hline \multicolumn{6}{|l|}{ Year of event (per event) } \\
\hline Congestive heart failure & $\$ 29,393$ & $\$ 23,514$ & $\$ 35,271$ & Normal & Ward 31,32 \\
\hline Ischemic heart disease & $\$ 26,483$ & $\$ 21,186$ & $\$ 31,780$ & Normal & Ward 31,32 \\
\hline Myocardial infarction & $\$ 69,832$ & $\$ 55,866$ & $\$ 83,799$ & Normal & Ward 31,32 \\
\hline Stroke & $\$ 52,108$ & $\$ 41,687$ & $\$ 62,530$ & Normal & Ward $^{31,32}$ \\
\hline Foot ulcer & $\$ 2,656$ & $\$ 2,125$ & $\$ 3,187$ & Normal & Ward $^{31,32}$ \\
\hline Amputation & $\$ 11,185$ & $\$ 8,948$ & $\$ 13,422$ & Normal & Ward 31,32 \\
\hline \multicolumn{5}{|l|}{ Hypoglycemia } & Ward $^{31,32}$ \\
\hline Requiring hospitalization & $\$ 20,386$ & $\$ 16,309$ & $\$ 24,463$ & Normal & Ward 31,32 \\
\hline Requiring ED visit & $\$ 1,622$ & $\$ 1,298$ & $\$ 1,946$ & Normal & Ward 31,32 \\
\hline Requiring glucagon injection & $\$ 218$ & $\$ 174$ & $\$ 261$ & Normal & Ward 31,32 \\
\hline
\end{tabular}




\section{TABLE 1 Model Parameters (continued)}

\begin{tabular}{|c|c|c|c|c|c|}
\hline & Base Case & Lower & Upper & $\begin{array}{l}\text { Probabilistic } \\
\text { Distribution }\end{array}$ & Source \\
\hline \multicolumn{6}{|l|}{ Clinical Costs } \\
\hline \multicolumn{6}{|c|}{ History of complication (per year) } \\
\hline Congestive heart failure & $\$ 2,356$ & $\$ 1,884$ & $\$ 2,827$ & Normal & Ward 31,32 \\
\hline Ischemic heart disease & $\$ 2,356$ & $\$ 1,884$ & $\$ 2,827$ & Normal & Ward 31,32 \\
\hline Myocardial infarction & $\$ 2,356$ & $\$ 1,884$ & $\$ 2,827$ & Normal & Ward $^{31,32}$ \\
\hline Stroke & $\$ 19,226$ & $\$ 15,381$ & $\$ 23,071$ & Normal & Ward $^{31,32}$ \\
\hline Blindness & $\$ 3,541$ & $\$ 2,833$ & $\$ 4,249$ & Normal & Ward $^{31,32}$ \\
\hline Renal disease & $\$ 88,723$ & $\$ 70,978$ & $\$ 106,467$ & Normal & Ward ${ }^{31,32}$ \\
\hline \multicolumn{6}{|l|}{ Treatment Costs } \\
\hline \multicolumn{6}{|c|}{ Add-on treatments (annual cost) } \\
\hline Oral semaglutide & $\$ 6,103$ & $\$ 4,883$ & $\$ 7,324$ & Normal & SSR Health, WAC 29,30 \\
\hline Sitagliptin (Januvia) & $\$ 1,360$ & $\$ 1,088$ & $\$ 1,632$ & Normal & SSR Health, WAC ${ }^{29,30}$ \\
\hline Empagliflozin (Jardiance) & $\$ 1,965$ & $\$ 1,572$ & $\$ 2,358$ & Normal & SSR Health, WAC 29,30 \\
\hline Liraglutide (Victoza) & $\$ 5,149$ & $\$ 4,120$ & $\$ 6,179$ & Normal & SSR Health, WAC 29,30 \\
\hline \multicolumn{6}{|l|}{ Background treatments } \\
\hline Metformin & $\$ 194$ & $\$ 155$ & $\$ 233$ & Normal & Laiteerapong ${ }^{18}$ \\
\hline Sulfonylurea & $\$ 86$ & $\$ 69$ & $\$ 103$ & Normal & Laiteerapong ${ }^{18}$ \\
\hline \multicolumn{6}{|l|}{ Insulin cost/unit } \\
\hline Basal & $\$ 0.22$ & $\$ 0.17$ & $\$ 0.26$ & Normal & SSR Health ${ }^{29}$ \\
\hline Bolus & $\$ 0.28$ & $\$ 0.23$ & $\$ 0.34$ & Normal & SSR Health 29 \\
\hline Premix & $\$ 0.14$ & $\$ 0.11$ & $\$ 0.17$ & Normal & SSR Health ${ }^{29}$ \\
\hline
\end{tabular}

OM2 estimated baseline rates, which were calculated from each NHANES person's individual characteristics, to derive the MACE, HF, and nephropathy (ESRD risk equation) outcome rates for the add-on treatments.

Specifically, the NMA-derived HRs for oral semaglutide versus placebo were applied to the baseline UKPDS OM2 equations to derive rates for oral semaglutide, while the rates for empagliflozin, liraglutide, and sitagliptin were derived by first applying the oral semaglutide versus placebo HRs, then applying each comparators' HR versus oral semaglutide (full league table of NMA results are shown in Supplementary Table 2, available in online article).

We made several assumptions regarding our application of NMA HRs to the UKPDS OM2 equations. First, we assumed that the incremental rates of MACE, HF, and kidney function decline were independent of individual characteristics, including A1c control, given that contemporary clinical trials have demonstrated an independent relationship between MACE and renal failure beyond the health effects based on changes in A1c. Second, we assumed that MACE and HF risk were independent due to the UKPDS risk equations; however, we acknowledge that these 2 conditions frequently co-occur. Third, we assumed the HR adjustments were maintained over each person's lifetime, given that long-term effectiveness is currently unknown. Fourth, we assumed relative risks between treatment regimens were uniformly distributed across all people with T2DM, not just those in the PIONEER program, allowing us to apply the trial-derived HRs to the non-trial NHANES population, since we did not have access to PIONEER patient-level data. Finally, we conservatively assumed no effect on nephropathy for sitagliptin because no data have been published for this outcome. No HR calibration was used for the background treatment comparator.

\section{UTILITIES}

We used consistent health state utility values across treatments evaluated in the model. Each person's specific utility 
value for a given year was derived from a baseline utility and applicable regression coefficients as estimated by Shao et al. (2019) for (a) complications in the year of an event, (b) history of complications, and (c) demographic characteristics. ${ }^{26}$ We added missing regression coefficients for foot ulcer and amputation events by assuming values from a recent diabetes utility study by Sullivan and Ghushchyan (2016). ${ }^{27}$ In Shao et al., the Health Utilities Index Mark 3 was used to measure heath utility in a sample of 8,713 people from the Action to Control Cardiovascular Risk in Diabetes (ACCORD) trial of people with T2DM with high risk of cardiovascular disease. ${ }^{26}$ Sullivan and Ghushchyan mapped EQ-5D-3L questionnaire responses to the Short Form-12 health survey responses of 20,705 individuals with diabetes (types 1 and 2) in the Medical Expenditure Panel Survey (MEPS) database from 2000 to $2011 .{ }^{27}$ Finally, we modeled an annual disutility for daily injection of insulin (for people who discontinue treatment) and liraglutide based on Boye et al. (2011), who used standard gamble interviews of people with T2DM in Scotland to estimate the utility values for injection-related attributes. ${ }^{28}$

\section{COSTS}

We obtained net drug pricing estimates from SSR Health, which are net of discounts, rebates, concessions to wholesalers and distributors, and patient assistance programs, to derive a net price. ${ }^{29}$ We estimated net prices by comparing recent 4-quarter averages (i.e., first quarter of 2019 through fourth quarter of 2019) of net prices and wholesale acquisition cost (WAC) per unit to arrive at a mean discount from WAC for the drug. ${ }^{30}$ We applied this average discount to the current WAC (accessed May 2020) to arrive at an estimated net price per unit. For oral semaglutide, we applied the average discount from WAC for injectable semaglutide to arrive at an estimated net price.

We obtained costs for T2DM-related complications and hypoglycemia from Ward et al. (2014), who estimated direct medical costs from data sources that included inpatient and emergency department databases, national physician and laboratory fee schedules, government reports, and published literature. ${ }^{31}$ Complication costs in the year of the event reflect acute care and any subsequent care provided in the first year; history of state costs reflect annual resource use for the ongoing management of complications in subsequent years. ${ }^{31}$ Costs were assessed from the perspective of a comprehensive U.S. health care payer, including patient cost sharing, and were inflated to May 2020 U.S. dollars. ${ }^{32}$ Other health care costs related to diabetes monitoring were also included.

\section{ADDITIONAL MODULES}

We applied pooled estimates of treatment discontinuation due to adverse events in the first model cycle. People discontinuing their primary modeled treatment were assumed to transition to insulin therapy to facilitate head-to-head comparator evaluations, as opposed to evaluating differences in multiple potential treatment pathways. ${ }^{22}$ Insulin treatment costs were based on a multivariate prediction model for estimating long-term A1c change, weight change, and hypoglycemic events associated with insulin rescue medication. ${ }^{33}$ After the first cycle, clinical characteristics for before and after insulin status were modeled using the multivariate prediction model's equations for A1c and weight change, which then influenced the UKPDS OM2 complication risk equations for those people. ${ }^{33}$

Mild, moderate, and severe hypoglycemia were modeled in subsequent cycles based on the previous UKPDS OM2 adaptation from Laiteerapong et al. (2018)..$^{18}$ People not yet receiving insulin were assumed to have a $5 \%$ probability for a severe hypoglycemic event and a 33\% probability for a mild or moderate event each year. People receiving insulin were assumed to have a $21 \%$ probability of a severe hypoglycemic event and a $52 \%$ probability of a mild or moderate hypoglycemic event each year. We assumed no more than 1 mild or moderate hypoglycemic event and 1 severe hypoglycemic event per year but allowed multiple hypoglycemic events during each lifetime.

Finally, the UKPDS OM2 equations have coefficients for atrial fibrillation and peripheral artery disease but the NHANES dataset did not provide this information. Therefore, we used age-based cumulative incidence estimates from the U.S. population and (for atrial fibrillation) relative risk estimates based on individuals' A1c to simulate these characteristics before each microsimulation. ${ }^{34,35}$ Peripheral artery disease and atrial fibrillation prevalence were modeled independent of existing person-level characteristics.

\section{MODEL ANALYSIS}

The model estimated the lifetime average survival, qualityadjusted survival, number of T2DM complications, and drug and T2DM complication costs for the 362 included NHANES individuals. Unlike a traditional Markov cohort model with deterministic base case results, the base-case result for each model outcome was the average of all simulations, in this case 15,000 microsimulations per person $(5,430,000$ total simulations). Time spent in each T2DM health state was summed to provide estimates of life expectancy and quality-adjusted life expectancy. We calculated the incremental costs, incremental life-years (LYs), incremental qualityadjusted life-years (QALYs), and incremental cost per LY 


\section{FIGURE 2 Results of Network Meta-Analysis}

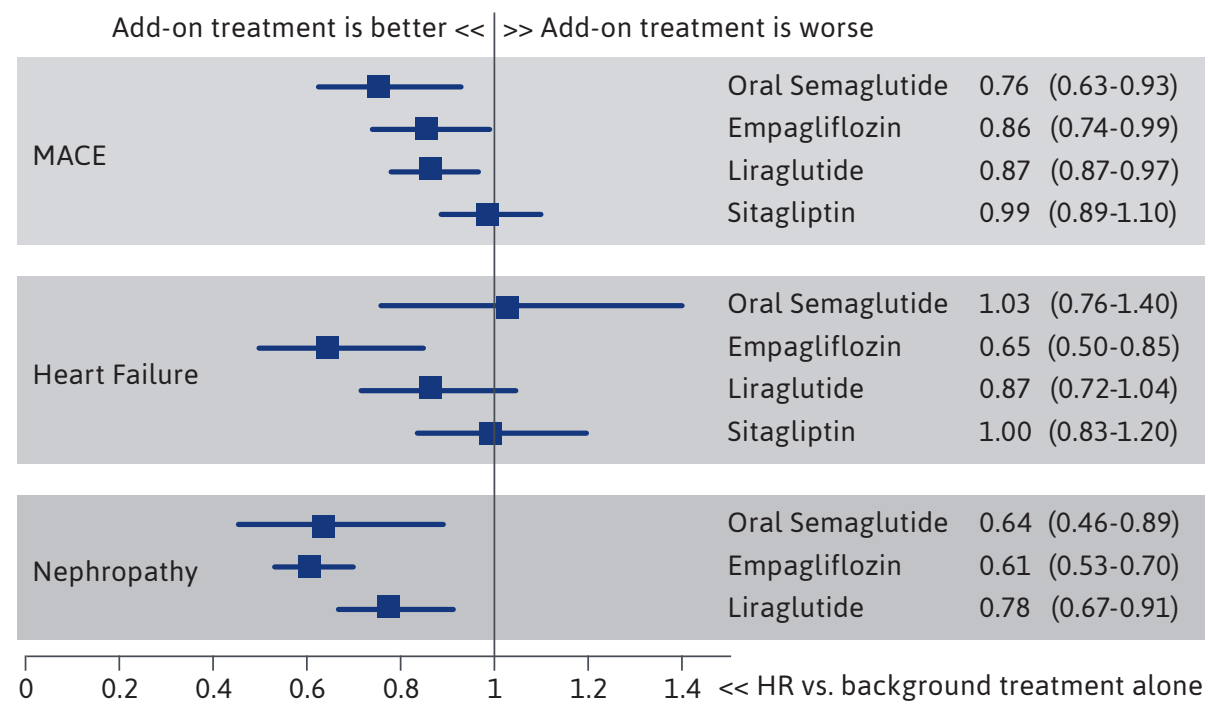

$H R=$ hazard ratio; $M A C E=$ major adverse cardiovascular event

gained and per QALY gained for oral semaglutide versus each comparator intervention. We also calculated the annual cost of oral semaglutide that was necessary to meet willingness-topay thresholds of $\$ 50,000$ per QALY, $\$ 100,000$ per QALY, and $\$ 150,000$ per QALY against each comparator.

\section{SCENARIO AND SENSITIVITY ANALYSES}

We performed scenario analyses to explore the effect of our model assumptions. First, we modeled a modified societal perspective by adding age-specific annual estimates of indirect costs related to the burden of diabetes, accounting for age and work status, in the following categories: absenteeism, presenteeism, inability to work, and decreased productivity for those not in the workforce. These 4 categories of indirect cost were abstracted from a previously published analysis that produced estimates from the National Health Interview Survey and applied as summary estimates separately for people aged 18-44 years $(\$ 5,580 /$ year), aged $45-64$ years (\$5,320/year), and aged 65 years and above $(\$ 1,480 /$ year $){ }^{1}$

Second, we modeled gradual decreases in oral semaglutide's longterm effectiveness on MACE and renal outcomes over time by annually increasing the MACE and nephropathy risk reductions starting in year 2 of the model. We created scenarios specific to MACE and renal disease that applied a $10 \%$ relative adjustment in the incremental effectiveness per year until the HRs reached 1.00 (no incremental effectiveness versus background treatment alone).

Third, we performed a threshold analysis by systematically altering the price of oral semaglutide to estimate the maximum prices that would correspond to common willingness-to-pay thresholds versus each comparator.

We performed one-way sensitivity analyses to identify the key drivers of model outcomes, using available measures of parameter uncertainty (i.e., standard errors) or reasonable ranges for each model parameter described above (see Table 1 for the ranges and distributions used in the sensitivity analyses). In order to efficiently operationalize the one-way sensitivity analysis within the framework of the person-level Monte Carlo microsimulation, we used a fixed random seed set and performed a single UKPDS equation simulation for each of 362 NHANES people for each parameter's low and high value in order to produce an estimate of uncertainty for each high and low value. Therefore, each low and high value represents the average effect over 362 individual simulations.

Probabilistic sensitivity analysis was performed in conjunction with the primary analysis by jointly varying all model parameters, accounting for sampling uncertainty and parameter uncertainty over 15,000 individual simulations for each person, then calculating $95 \%$ credible range (CR) estimates for each outcome based on the results. ${ }^{36}$

\section{Results}

\section{EVIDENCE SYNTHESIS RESULTS}

Results from our NMA showed that semaglutide (oral and injectable) significantly reduced the risk for 3-point MACE compared with background treatment alone $(\mathrm{HR}=0.76 ; 95 \%$ credible interval $[\mathrm{CrI}]=0.63-0.93)$ and sitagliptin $\quad(\mathrm{HR}=0.77 ; 95 \% \mathrm{CrI}=0.61-$ 0.96; Figure 2 and Supplementary Table 2, available in online article). Results also showed a nonsignificant risk reduction of semaglutide for MACE compared with empagliflozin $(\mathrm{HR}=0.88 ; 95 \% \mathrm{CrI}=0.69-1.13)$ and liraglutide (HR=0.87; 95\% CrI $=0.70-1.09$ ). Empagliflozin significantly reduced the risk for HF compared with semaglutide $(\mathrm{HR}=0.63 ; 95 \% \mathrm{CrI}=0.42-0.95)$. There were no significant differences 


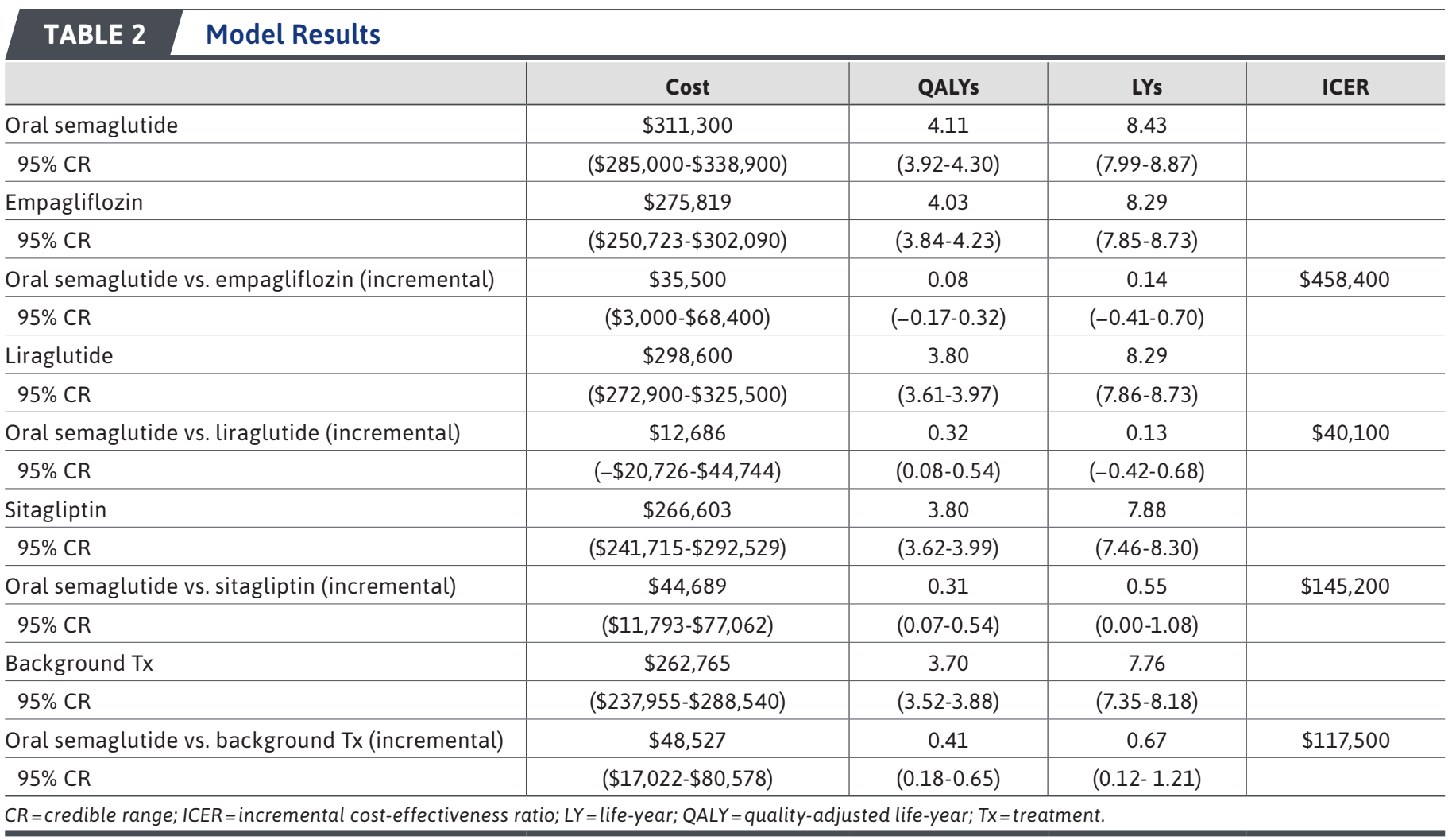

with semaglutide and any of the active comparators of interest on nephropathy.

\section{MICROSIMULATION}

The lifetime mean total cost for people treated with oral semaglutide was $\$ 311,300 \quad(95 \% \quad C R=\$ 285,000-\$ 338,900)$ and costs for the other comparators ranged from $\$ 262,800$ (background treatment alone) to $\$ 298,600$ (liraglutide; Table 2 and Supplementary Table 3, available in online article). Oral semaglutide resulted in the fewest MACE (0.643 per person vs. 0.663 [liraglutide] to 0.714 [background treatment alone]), including the fewest cardiovascular deaths (0.073 per person vs. 0.090 [empagliflozin] to 0.095 [background treatment alone]). Among the 5 modeled treatment strategies, oral semaglutide had the highest LYs gained (8.43 [95\% CR=7.99-8.87] vs. 7.76 [background treatment alone] to 8.29 [empagliflozin and liraglutide]), as well as QALYs gained (4.11 [95\% CR=3.92-4.30] vs. 3.70 [background treatment alone] to 4.03 [empagliflozin]).

Oral semaglutide was cost-effective compared with liraglutide, with an ICER of $\$ 40,100$, and was between $\$ 100,000$ and $\$ 150,000$ per QALY when compared with background treatment alone $(\mathrm{ICER}=\$ 117,500 / \mathrm{QALY})$ and sitagliptin $($ ICER $=\$ 145,219 /$ QALY; Table 2 and Supplementary Table 4, available in online article). The ICER for oral semaglutide compared with empagliflozin was approximately $\$ 458,400$ per QALY. The estimated costs per MACE avoided for oral semaglutide were $\$ 782,500$ versus sitagliptin, $\$ 997,600$ versus empagliflozin, $\$ 650,700$ versus liraglutide, and $\$ 685,100$ versus background treatment alone.

\section{SCENARIO ANALYSES}

Adding productivity costs to the model resulted in similar ICERs for oral semaglutide versus each of the comparators as the base case without these societal costs (Table 3). Small differences in ICERs compared with the base case were due to incremental societal costs that largely canceled out between comparators except for incremental survival differences. When we gradually reduced the efficacy of oral semaglutide for MACE and renal outcomes by $10 \%$ per year, this increased the lifetime incidence of MACE and renal outcomes, leading to increased cost and decreased LYs and QALYs for oral semaglutide. 


\section{TABLE 3 Results of Scenario Analyses}

\begin{tabular}{|c|c|c|c|c|}
\hline Comparison & Scenario & Incremental Cost & Incremental QALYs & ICER \\
\hline \multirow{7}{*}{$\begin{array}{l}\text { Oral semaglutide } \\
\text { vs. empagliflozin }\end{array}$} & Base case & $\$ 35,500$ & 0.08 & $\$ 458,400$ \\
\hline & $\$ 50 K$ threshold annual cost $=\$ 5,487$ & $\$ 3,900$ & 0.08 & $\$ 50,000$ \\
\hline & $\$ 100 \mathrm{~K}$ threshold annual cost $=\$ 5,562$ & $\$ 7,700$ & 0.08 & $\$ 100,000$ \\
\hline & $\$ 150 \mathrm{~K}$ threshold annual cost $=\$ 5,636$ & $\$ 11,600$ & 0.08 & $\$ 150,000$ \\
\hline & Modified societal perspective & $\$ 36,700$ & 0.08 & $\$ 459,300$ \\
\hline & $10 \%$ annual OS MACE efficacy decline & $\$ 33,600$ & 0.02 & $\$ 1,521,700$ \\
\hline & $10 \%$ annual OS nephropathy efficacy decline & $\$ 37,200$ & 0.06 & $\$ 618,900$ \\
\hline \multirow{7}{*}{$\begin{array}{l}\text { Oral semaglutide } \\
\text { vs. liraglutide }\end{array}$} & Base case & $\$ 12,700$ & 0.32 & $\$ 40,100$ \\
\hline & $\$ 50 \mathrm{~K}$ threshold annual cost $=\$ 6,170$ & $\$ 15,800$ & 0.32 & $\$ 50,000$ \\
\hline & $\$ 100 \mathrm{~K}$ threshold annual cost $=\$ 6,478$ & $\$ 31,600$ & 0.32 & $\$ 100,000$ \\
\hline & $\$ 150 \mathrm{~K}$ threshold annual cost $=\$ 6,787$ & $\$ 47,400$ & 0.32 & $\$ 150,000$ \\
\hline & Modified societal perspective & $\$ 13,100$ & 0.32 & $\$ 41,400$ \\
\hline & $10 \%$ annual OS MACE efficacy decline & $\$ 10,800$ & 0.26 & $\$ 40,800$ \\
\hline & $10 \%$ annual OS nephropathy efficacy decline & $\$ 14,000$ & 0.30 & $\$ 47,200$ \\
\hline \multirow{7}{*}{$\begin{array}{l}\text { Oral semaglutide } \\
\text { vs. sitagliptin }\end{array}$} & Base case & $\$ 44,700$ & 0.31 & $\$ 145,200$ \\
\hline & $\$ 50 K$ threshold annual cost $=\$ 5,533$ & $\$ 15,400$ & 0.31 & $\$ 50,000$ \\
\hline & $\$ 100 K$ threshold annual cost $=\$ 5,834$ & $\$ 30,800$ & 0.31 & $\$ 100,000$ \\
\hline & $\$ 150 K$ threshold annual cost $=\$ 6,134$ & $\$ 46,200$ & 0.31 & $\$ 150,000$ \\
\hline & Modified societal perspective & $\$ 46,000$ & 0.31 & $\$ 149,500$ \\
\hline & $10 \%$ annual OS MACE efficacy decline & $\$ 43,600$ & 0.26 & $\$ 170,200$ \\
\hline & $10 \%$ annual OS nephropathy efficacy decline & $\$ 46,000$ & 0.29 & $\$ 158,000$ \\
\hline \multirow{7}{*}{$\begin{array}{l}\text { Oral semaglutide } \\
\text { vs. background } \\
\text { treatment alone }\end{array}$} & Base case & $\$ 48,500$ & 0.41 & $\$ 117,500$ \\
\hline & $\$ 50 \mathrm{~K}$ threshold annual cost $=\$ 5,562$ & $\$ 20,700$ & 0.41 & $\$ 50,000$ \\
\hline & $\$ 100 \mathrm{~K}$ threshold annual cost $=\$ 5,965$ & $\$ 41,300$ & 0.41 & $\$ 100,000$ \\
\hline & $\$ 150 \mathrm{~K}$ threshold annual cost $=\$ 6,369$ & $\$ 62,000$ & 0.41 & $\$ 150,000$ \\
\hline & Modified societal perspective & $\$ 50,100$ & 0.41 & $\$ 121,300$ \\
\hline & $10 \%$ annual OS MACE efficacy decline & $\$ 47,000$ & 0.36 & $\$ 129,900$ \\
\hline & $10 \%$ annual OS nephropathy efficacy decline & $\$ 50,000$ & 0.40 & $\$ 125,700$ \\
\hline
\end{tabular}

ICER = incremental cost-effectiveness ratio; $M A C E=$ major adverse cardiovascular event; $O S=$ oral semaglutide; $Q A L Y=$ quality-adjusted life-year.

In general, ICERs tended to increase for oral semaglutide versus each comparator. The annual cost of oral semaglutide needed to reach a cost-effectiveness threshold of $\$ 50,000$ per QALY gained ranged from $\$ 5,487$ (vs. empagliflozin; 95\% $\mathrm{CR}=\$ 4,307-\$ 6,726)$ to $\$ 6,170$ (vs. liraglutide; $95 \% \mathrm{CR}=\$ 4,834-\$ 7,577)$. The annual cost needed to reach a threshold of $\$ 100,000$ per QALY gained ranged from $\$ 5,562$ (vs. empagliflozin; $95 \% \mathrm{CR}=\$ 4,376-\$ 6,812$ ) to $\$ 6,478$ (vs. liraglutide; 95\% $\mathrm{C}=\$ 5,096-\$ 7,937$; see Table 3 and Supplementary Table 4 , available in online article, for complete scenario results).

\section{SENSITIVITY ANALYSES}

Across comparisons, the parameters with the greatest effect on incremental cost were MACE HRs, add-on treatment costs, and HRs for HF and nephropathy (Supplementary Figure 1, available in online article). The parameters with the greatest effect on incremental QALYs were MACE HRs, short-term trial-derived changes in A1c, HRs for $\mathrm{HF}$ and nephropathy, the annual disutility for injectable treatments (for the comparison vs. liraglutide), and utility parameters (in the comparison vs. background treatment alone; Supplementary Figure 2, available in online article). 
In the probabilistic sensitivity analysis, oral semaglutide was predicted to be cost-effective compared with liraglutide at thresholds above $\$ 100,000$ per QALY and to have a more than a $50 \%$ chance of being cost-effective against sitagliptin or background treatment alone at a threshold of $\$ 150,000$ per QALY or higher. However, even at a threshold of $\$ 250,000$ per QALY, oral semaglutide had only a $27 \%$ chance of being cost-effective compared with empagliflozin (Supplementary Figure 3, available in online article).

\section{Discussion}

We developed a patient-level microsimulation (adapted from previous models ${ }^{18,19}$ ) to compare the clinical and economic impact of 5 different treatment strategies for people with T2DM. Oral semaglutide as an add-on therapy to background antihyperglycemic treatment was estimated to produce incremental benefits in MACE avoided and incremental QALYs compared with background antihyperglycemic treatment alone. Oral semaglutide was estimated to result in better outcomes than background treatment alone or sitagliptin and similar outcomes to liraglutide or empagliflozin. At an estimated net price of $\$ 6,103$ per year, oral semaglutide was estimated to be cost-effective compared with liraglutide and to be moderately cost-effective versus sitagliptin and background therapy alone, with ICERs between $\$ 100,000$ and $\$ 150,000$ per QALY. Oral semaglutide was not found to be cost-effective compared with empagliflozin.

The primary justification for adding oral semaglutide to a health plan formulary is the advantage of having an oral option for GLP-1 receptor agonist therapy. Many people with T2DM are hesitant to move to treatment with injectable medications. However, many people cannot achieve their target A1c using lifestyle modification and other oral medications alone. Oral semaglutide therefore is likely to allow many people to remain on oral treatment who would otherwise require either an injectable GLP-1 receptor agonist or insulin.

Our base-case results represent averages over sufficient simulations to achieve statistical convergence. Result uncertainties are reflected in statistical variance in the model input parameters and risk equations, as shown in the probabilistic sensitivity analyses, and in the additional uncertainties from the NMA caused by concerns about whether effect modification could result from differences in the underlying cardiovascular outcomes trials. In addition, all results assume the same net price discount from WAC for oral semaglutide as for injectable semaglutide. If the actual net price is different, these results would change.

\section{LIMITATIONS}

As with all decision modeling exercises, the limitations of our approach and assumptions should be considered when interpreting these findings. The principal limitation of our model is the complexity of T2DM, its comorbidities, and its person-specific clinical management. Because it is difficult to expect regression equations to reliably predict any one person's actual outcomes, we undertook a large number of sensitivity and scenario analyses to avoid depending on a single deterministic output.

Second, the cardiovascular and renal outcome estimates for our model could only be estimated from indirect treatment comparisons (by NMA) that are potentially susceptible to effect modification. We also assumed that the cardiovascular benefits observed in the trials that targeted MACE as the primary outcomes remained constant for each person's lifetime. With a lack of data on longer term follow-up for these events or real-world evidence of adherence and its relationship with such benefits, we were required to make this assumption. Scenario analyses showed that a gradual decrease in long-term efficacy led to increased cost and decreased LYs and QALYs for oral semaglutide, and ICERs tended to increase versus each comparator.

Third, the utility values for events modeled from the risk equations were drawn from 2 sources (and different instruments) because of the lack of a single comprehensive source of health-related quality of life inputs.

Finally, people with T2DM are treated based on clinical guidelines, which had been muted for this modeling exercise. We assumed that all persons discontinuing their initial treatment received insulin in order to provide direct head-to-head estimates of value for those initial treatment decisions. However, individuals would likely experience a cascade of different treatments upon discontinuation, which could have different costs and outcomes for that person than what were modeled.

\section{Conclusions}

We found that at its estimated net price, oral semaglutide is likely cost-effective versus liraglutide, is moderately costeffective compared with sitagliptin and background therapy alone, but is not cost-effective compared with empagliflozin. 


\section{DISCLOSURES}

Funding for this study was provided by the Institute for Clinical and Economic Review, an independent organization that evaluates the evidence on the value of health care interventions. ICER reports grants from Laura and John Arnold Foundation, California Health Care Foundation, Harvard Pilgrim Health Care, and Kaiser Foundation Health Plan. ICER's annual policy summit is supported by dues from AbbVie, Aetna, America's Health Insurance Plans, Anthem, Alnylam, AstraZeneca, Biogen, Blue Shield of CA, Cambia Health Services, CVS, Editas, Evolve Pharmacy, Express Scripts, Genentech/Roche, GlaxoSmithKline, Harvard Pilgrim, Health Care Service Corporation, Health Partners, Humana, Johnson \& Johnson (Janssen), Kaiser Permanente, LEO Pharma, Mallinckrodt, Merck, Novartis, National Pharmaceutical Council, Premera, Prime Therapeutics, Regeneron, Sanofi, Spark Therapeutics, uniQure, and United Healthcare.

Rind, Fazioli, Chapman, and Pearson are employed by ICER. Guzauskas and Hansen have nothing to disclose.

Study results were presented at the New England Comparative Effectiveness Public Advisory Council (New England CEPAC), November 14, 2019, at Brown University, Providence, RI.

\section{REFERENCES}

1. Dall TM, Yang W, Gillespie K, et al. The economic burden of elevated blood glucose levels in 2017: diagnosed and undiagnosed diabetes, gestational diabetes mellitus, and prediabetes. Diabetes Care. 2019;42(9):1661-68. doi:10.2337/ dc18-1226

2. Davies MJ, D'Alessio DA, Fradkin J, et al. Management of hyperglycemia in type 2 diabetes, 2018. a consensus report by the American Diabetes Association (ADA) and the European Association for the Study of Diabetes (EASD). Diabetes Care. 2018;41(12):2669-701. doi:10.2337/ dci18-0033
3. McCulloch DK. Glycemic control and vascular complications in type 2 diabetes mellitus. In: Mulder JE, ed. UpToDate. March 10, 2020. Retrieved March 2, 2021. Accessed July 7, 2020. https://www.uptodate.com/contents/ glycemic-control-and-vascular-complications-in-type-2-diabetes-mellitus

4. American Diabetes Association. The cost of diabetes. Retrieved May 20, 2020. Accessed March 2, 2021. https://www. diabetes.org/resources/statistics/ cost-diabetes

5. Cohen RA, Cha AE. Strategies used by adults with diagnosed diabetes to reduce their prescription drug costs, 2017-2018. NCHS Data Brief. 2019;(349):1-8.

6. American Diabetes Association. 9. Pharmacologic approaches to glycemic treatment: standards of medical care in diabetes-2019. Diabetes Care. 2019;42(Suppl 1):S90-S102. doi:10.2337/ dc19-S009

7. Novo Nordisk. Ozempic ${ }^{\circledR}$ provided greater weight reductions for adults with a baseline BMI of or above $25 \mathrm{~kg} / \mathrm{m} 2$ than those with lower baseline BMI $<25 \mathrm{~kg} / \mathrm{m} 2$, in a SUSTAIN 7 post-hoc analysis. Press release. June 23, 2018. Accessed March 10, 2021. https://www.novonordisk. com/content/nncorp/global/en/newsand-media/news-and-ir-materials / news-details.html?id=565

8. Novo Nordisk. Novo Nordisk files for U.S. FDA approval of oral semaglutide for blood sugar control and cardiovascular risk reduction in adults with type 2 diabetes. Press release. March 20, 2019. Accessed March 2, 2021. https:// www.prnewswire.com/news-releases/ novo-nordisk-files-for-us-fda-approvalof-oral-semaglutide-for-blood-sugarcontrol-and-cardiovascular-riskreduction-in-adults-with-type-2-diabetes-300815668.html

9. Centers for Disease Control and Prevention, National Center for Health Statistics (NCHS). National Health and Nutrition Examination Survey Data. 20152016. Retrieved August 20, 2019. Accessed March 2, 2021. https://wwwn.cdc.gov/ nchs/nhanes/ContinuousNhanes/ Default.aspx?BeginYear $=2015$
10. Rind D, Guzauskas G, Fazioli K, et al. Oral semaglutide for type 2 diabetes: effectiveness and value. Final evidence report. December 9, 2019. Accessed March 10, 2021. https://icer.org/wp-content/ uploads/2020/10/ICER_Diabetes_FinalEvidence-Report 120919-1.pdf

11. Husain M, Birkenfeld AL, Donsmark M, et al. Oral semaglutide and cardiovascular outcomes in patients with type 2 diabetes. New Engl J Med. 2019;381(9):841-51. doi:10.1056/NEJMoa1901118

12. Marso SP, Bain SC, Consoli A, et al. Semaglutide and cardiovascular outcomes in patients with type 2 diabetes. New Engl J Med. 2016;375(19):1834-44. doi:10.1056/ NEJMoa1607141

13. Viechtbauer W. Conducting meta-analyses in R with the metafor package. J Stat Soft. 2010;36(3):1-48.

14. van Valkenhoef G, Kuiper J. gemtc: Network Meta-Analysis Using Bayesian Methods. R package version 0.8-2. 2016. Accessed March 10, 2021. https://cran.rproject.org/web/packages/gemtc/index. $\underline{\mathrm{html}}$

15. Green JB, Bethel MA, Armstrong PW, et al. Effect of sitagliptin on cardiovascular outcomes in type 2 diabetes. New Engl J Med. 2015;373(3):232-42. doi:10.1056/ NEJMoa1501352

16. Marso SP, Daniels GH, Brown-Frandsen K, et al. Liraglutide and cardiovascular outcomes in type 2 diabetes. New Engl J Med. 2016;375(4): 311-22. doi:10.1056/NEJMoa1603827

17. Zinman B, Wanner C, Lachin JM, et al. Empagliflozin, cardiovascular outcomes, and mortality in type 2 diabetes. New Engl J Med. 2015;373(22):2117-28. doi:10.1056/ NEJMoa1504720

18. Laiteerapong N, Cooper JM, Skandari MR, et al. Individualized glycemic control for u.s. adults with type 2 diabetes: a cost-effectiveness analysis. Ann Intern Med. 2018;168(3):170-78. doi:10.7326/M17-0537 
19. Hayes AJ, Leal J, Gray AM, Holman RR, Clarke PM. UKPDS outcomes model 2: a new version of a model to simulate lifetime health outcomes of patients with type 2 diabetes mellitus using data from the 30 year United Kingdom Prospective Diabetes Study: UKPDS 82. Diabetologia. 2013;56(9):1925-33. doi:10.1007/

s00125-013-2940-y

20. Lundqvist A, Steen Carlsson K, Johansen P, Andersson E, Willis M. Validation of the IHE cohort model of type 2 diabetes and the impact of choice of macrovascular risk equations. PLoS One. 2014;9(10):e110235. doi:10.1371/journal. pone.0110235

21. McEwan P, Foos V, Palmer JL, Lamotte M, Lloyd A, Grant D. Validation of the IMS CORE Diabetes Model. Value Health. 2014;17(6):714-24. doi:10.1016/j. jval.2014.07.007

22. Hirsch IB, Gaudiani LM. Using insulin to treat poorly controlled type 2 diabetes in 2020. JAMA. 2020;323(23):2419-20. doi:10.1001/jama.2020.1303

23. Pratley R, Amod A, Hoff ST, et al. Oral semaglutide versus subcutaneous liraglutide and placebo in type 2 diabetes (PIONEER 4): a randomised, double-blind, phase 3a trial. Lancet. 2019;394(10192): 39-50. doi:10.1016/s0140-6736(19)31271-1

24. Rodbard HW, Rosenstock J, Canani LH, et al. Oral semaglutide versus empagliflozin in patients with type 2 diabetes uncontrolled on metformin: the PIONEER 2 trial. Diabetes Care. 2019;42(12):2272-81. doi:10.2337/dc19-0883
25. Rosenstock J, Allison D, Birkenfeld AL, et al. Effect of additional oral semaglutide vs sitagliptin on glycated hemoglobin in adults with type 2 diabetes uncontrolled with metformin alone or with sulfonylurea: the PIONEER 3 randomized clinical trial. JAMA. 2019;321(15):1466-80. doi:10.1001/jama.2019.2942

26. Shao H, Yang S, Fonseca V, Stoecker C, Shi L. Estimating quality of life decrements due to diabetes complications in the United States: The Health Utility Index (HUI) Diabetes Complication Equation. Pharmacoeconomics. 2019;37(7):921-29. doi:10.1007/ s40273-019-00775-8

27. Sullivan PW, Ghushchyan VH. EQ-5D Scores for diabetes-related comorbidities. Value Health. 2016;19(8):1002-08. doi:10.1016/j.jval.2016.05.018

28. Boye KS, Matza LS, Walter KN, Van Brunt K, Palsgrove AC, Tynan A. Utilities and disutilities for attributes of injectable treatments for type 2 diabetes. Eur J Health Econ. 2011;12(3):219-30. doi:10.1007/s10198-010-0224-8

29. SSR Health. Database. 2019. Accessed March 10, 2021. https://www.ssrhealth. com/

30. IBM. IBM Micromedex Red Book Online. Database. Retrieved October 1, 2019. Accessed March 2, 2021. http:// www.micromedexsolutions.com

31. Ward A, Alvarez P, Vo L, Martin S. Direct medical costs of complications of diabetes in the United States: estimates for event-year and annual state costs (USD 2012). J Med Econ. 2014;17(3):176-83. doi:10.3111/13696998.2014.882843
32. U.S. Bureau of Labor Statistics. Medical care in U.S. city average, all urban consumers, not seasonally adjusted. Retrieved July 15, 2019. Accessed March 2, 2021. https://data.bls.gov/timeseries/ CUUR0000SAM

33. Willis M, Asseburg C, Nilsson A, Johnsson K, Kartman B. Multivariate prediction equations for hba1c lowering, weight change, and hypoglycemic events associated with insulin rescue medication in type 2 diabetes mellitus: informing economic modeling. Value Health. 2017;20(3):357-71. doi:10.1016/j. jval.2016.10.004

34. Allison MA, Ho E, Denenberg JO, et al. Ethnic-specific prevalence of peripheral arterial disease in the United States. Am J Prev Med. 2007;32(4):328-33. doi:10.1016/j. amepre.2006.12.010

35. Aune D, Feng T, Schlesinger S, Janszky I, Norat T, Riboli E. Diabetes mellitus, blood glucose and the risk of atrial fibrillation: A systematic review and meta-analysis of cohort studies. J Diabetes Complications. 2018;32(5):501-11. doi:10.1016/j.jdiacomp.2018.02.004

36. Dakin HA, Leal J, Briggs A, Clarke P, Holman RR, Gray A. Accurately reflecting uncertainty when using patient-level simulation models to extrapolate clinical trial data. Med Decis Making. 2020;40(4): 460-73. doi:10.1177/0272989x20916442 